\title{
Systemic sclerosis sine scleroderma: a case report of anterior uveitis
}

\author{
T. Borges, J. Vilaça, S. Ferreira, I. Chora, S. Silva, C. Dias \\ Internal Medicine Department, Centro Hospitalar São João, Al Prof Hernâni Monteiro, Porto, Portugal
}

\begin{abstract}
SUMMARY
Systemic sclerosis (SSc) sine scleroderma (ssSSc) is characterized by the absence of skin involvement, despite other manifestations of systemic sclerosis are present. It is not known whether sSSc represents a forme fruste of limited cutaneous SSc or a distinct entity, but the 2013 American College of Rheumatology/European League Against Rheumatism criteria for the classification of SSc have considered SSc without skin involvement to be a distinct subset.

The authors present the case of a 70-year old female that was referred for a consultation for Raynaud's phenomenon and a chronic anterior uveitis (CAU). She had a history of dysphagia, diffuse pulmonary emphysema and a biopsy-documented fibrosis of the upper lobes, and an idiopathic non-ischemic dilated cardiomyopathy with severe left ventricle systolic dysfunction and left bundle branch block. Anti-nuclear and anti-centromere antibodies were positive, while manometry revealed distal esophageal hypomotility. After establishing the diagnosis of ssSSc and starting immunosuppression, the ocular disease improved, while the lung and heart diseases remained stable. This case underlines that it is very important to suspect SSc when CAU is present and/or skin thickening is absent. To our knowledge, this is the first report of CAU in a patient with ssSSc.
\end{abstract}

Key words: Systemic sclerosis, Uveitis, Pulmonary fibrosis, Dilated cardiomyopathy.

Reumatismo, 2015; 67 (1): 21-25

\section{INTRODUCTION}

A mong connective tissue diseases, systemic sclerosis (SSc) has the highest disease-related mortality, with a median survival of 11 years after diagnosis (1). The 2013 American College of Rheumatology/ European League Against Rheumatism (ACR/EULAR) criteria for the classification of SSc consider that skin thickening of the fingers of both hands extending proximally to the metacarpophalangeal joints is enough to classify a patient as having SSc. However three subsets have been recognized: limited cutaneous SSc (lcSSc), diffuse cutaneous SSc (dcSSc) and SSc without skin involvement (2). The last, also known as systemic sclerosis sine scleroderma (ssSSc), is a variant without clinically detectable skin involvement, yet with visceral and immunological manifestations that are characteristic of SSc (3). In the registry of the German Network for SSc, ssSSc was found to be present in $1.5 \%$, supporting the concept of Poomorghim et al. who consider it a clinical variant of lcSSc, with similar characteristics and no specific antibody profile (4). Besides skin thickening, there were no significant differences in terms of internal organ involvement, laboratory values, serum autoantibodies or survival rate compared to lcSSc (5). Meanwhile, a Brazilian cohort of 79 ssSSc patients and the Spanish registry with 69 ssSSc patients suggested that more cardiac involvement and less telangiectasia would differentiate it from lcSSc, but a recent multicenter study of $27 \mathrm{ssSSc}$ patients suggested that it is probably a forme fruste of lcSSc, meaning that the absence of skin involvement may be ascribed to a misclassification due to early or subtle involvement $(3,6,7)$. It has been proposed that the term scleroderma be abandoned on the grounds that it is incorrectly related to the diagnosis of SSc, and be potentially replaced by designations such as morphea (localized scleroderma), lcSSc (previously reported as CREST syndrome), dcSSc and $\operatorname{ssSSc}(1)$.
Corresponding author:

Tiago Borges

Avenida da República 396 5F 4430-188

Vila Nova de Gaia, Portugal

E-mail: mtiago.b@hotmail.com 
Data for ocular involvement in SSc is scarce. It appears to be variable, affecting both anterior and posterior segments, the orbit and the extra-ocular muscles (8). Uveitis is a known complication of SSc in infants, but the relationship between the two disorders is not as consistent in adults (9).

\section{CASE REPORT}

A 70-year-old white female was referred for a consultation for Raynaud's phenomenon (RP) and bilateral idiopathic uveitis. RP had been present for several years, was triphasic and induced by exposure to cold. The patient had already been on systemic corticosteroids (deflazacort $60 \mathrm{mg} /$ day) for several months due to a chronic anterior uveitis (CAU), after a poor response to topic corticosteroids and ofloxacin. She had noticed a severe lack of vision when she first attended her ophthalmologist and had only a partial improvement after taking a systemic therapy. Bilateral phacoemulsification had been postponed until the stabilization of the ocular disease. There was no history of arthritis, inflammatory back pain, myalgias, skin changes and oral or genital ulcerations. Sarcoidosis, tuberculosis, toxoplasmosis and syphilis had already been excluded based on clinical and laboratory grounds.

The patient had a history of previous tobacco use (total of 20 pack-years) and was being followed by a pneumologist for a stage $\mathrm{C}$ chronic obstructive pulmonary disease (COPD), diagnosed five years before, when she presented with moderate dyspnea. Relevant diagnostic procedures performed during that period included a highresolution computed tomography (HRCT), which revealed not only a diffuse centrilobular pulmonary emphysema but also a fibrosis of the upper lobes, a transthoracic needle biopsy that was performed due to a suspicious lung node [excluding any malignancy but revealing an extensive fibrosis in the adjacent parenchyma compatible with nonspecific interstitial pneumonia (NSIP)] and pulmonary function tests showing a mixed pattern with low forced vital capacity $(78.5 \%)$ and forced expiratory volume (FEV1 45.6\%), and a low diffusing capacity of the lungs for carbon monoxide (DLCO/VA 18.5\%). A functional test had revealed a reduced capacity to cover a six-minute walk distance $(360 \mathrm{~m})$ and a high oxygen desaturation after exercise $(7 \%)$, although values were normal for gas exchanges at rest. She was also being followed by a cardiologist due to an idiopathic non-ischemic dilated cardiomyopathy with severe left ventricle systolic dysfunction (LVSD). Since she was classified as New York Heart Association functional class III after the optimization of the medical therapy and had a complete left bundle branch block with QRS of more than 150 $\mathrm{ms}$, she had been submitted to the implantation of a cardiac resynchronization therapy-implantable cardioverter defibrillator. The last echocardiography reported an estimated left ventricular ejection fraction of $20 \%$, also revealing aortic valve sclerosis without stenosis, hypertrabeculation of the lateral, inferior and posterior myocardial walls and thickening of the papillary muscles and tendinous chords, without indirect signs of pulmonary arterial hypertension (PAH). Other cardiovascular risk factors included systemic hypertension and dyslipidemia. She was being followed by a gastroenterologist for chronic gastritis and esophagitis, as she also complained of difficulty in swallowing, despite having been treated with a proton pump inhibitor for several months.

During the clinical examination, pulmonary auscultation revealed diffuse inspiratory rales. No skin thickening, telangiectasia, digital tip ulcers or fingertip pitting scars were present. Nifedipine was started and led to an improvement of RP. Nailfold videocapillaroscopy revealing an early pattern is shown in Figure 1. Anti-nuclear antibodies (ANA) were positive in high titer $(>1 / 1000)$; anti-centromere antibodies (ACA) were also positive. The remaining autoimmunity profile was negative including anti-topoisomerase, while blood counts were normal and blood chemistry revealed normal values for urea and creatinine, liver 


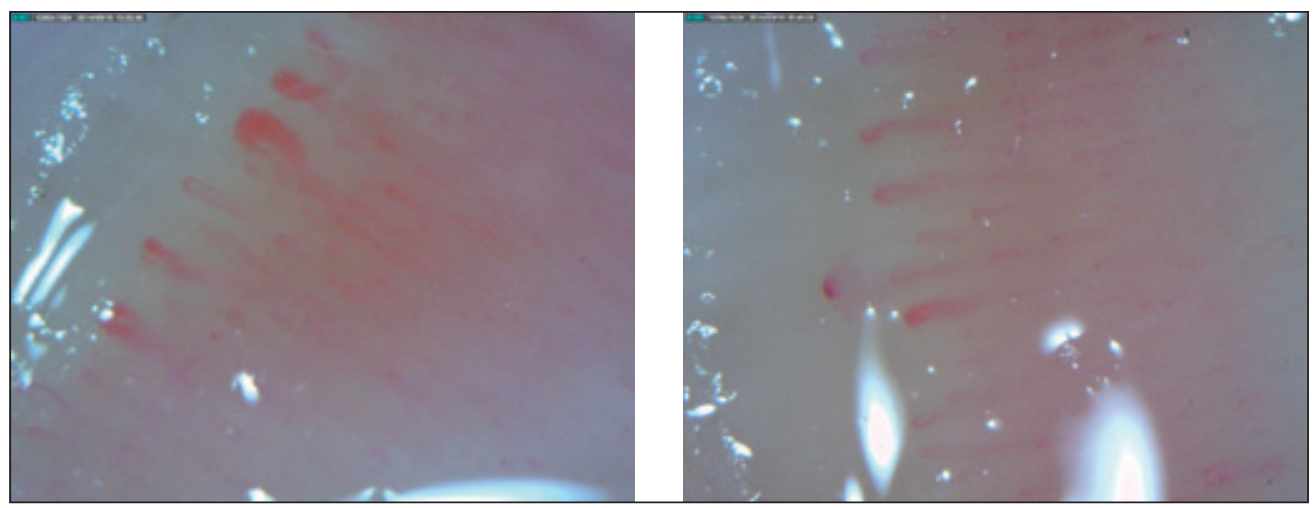

Figure 1 - Nailfold capillaroscopy revealing an early pattern with a relatively well preserved architecture, some megacapillaries (left) and few hemorrhages (right, arrow).

enzymes and acute-phase reactants including C-reactive protein $(1.8 \mathrm{mg} / \mathrm{L})$. Manometry revealed distal esophageal hypomotility and normal pressure in the esophageal sphincters, which were compatible with SSc. Immunosuppression was initiated. After three months, ocular disease had improved with a resolution of CAU, while the pulmonary and cardiac diseases were stable.

\section{DISCUSSION}

The patient fulfills the 2013 ACR/EULAR criteria for SSc: although she does not have skin thickening, abnormal nailfold capillaries ( 2 points), interstitial lung disease ( 2 points), RP (3 points) and SS-related autoantibodies (3 points) are sufficient. She also fulfills all the criteria defined by Poormoghim et al. for ssSSc diagnosis: absent skin thickening, RP, positive ANA, one visceral organ involvement (distal esophageal or small bowel hypomotility, pulmonary interstitial fibrosis, pulmonary hypertension, typical cardiac involvement or renal failure consistent with scleroderma renal crisis) and absence of another connective tissue disease or any other disease that explains the clinical picture (3). Even though emphysema and NSIP seem to coexist in her lungs, the latter seems to be caused by SSc, along with distal esophageal hypomotility and, possibly, idiopathic cardiomyopathy.

In general, ssSSc affects predominantly white women (4 to $5: 1$ ratio) in their middle age (mean of 50 years) $(6,10)$. Table I shows the prevalence of clinical features

Table I - Prevalence of some manifestations in systemic sclerosis sine scleroderma.

\begin{tabular}{|c|c|c|c|c|c|}
\hline & $\begin{array}{l}\text { Poormoghim et al., } \\
2000(5)(n=48)\end{array}$ & $\begin{array}{l}\text { Hunzelmann et al., } \\
2008(4)(n=22)\end{array}$ & $\begin{array}{l}\text { Siméon-Aznar et al., } \\
2012(7)(n=69)\end{array}$ & $\begin{array}{l}\text { Marangoni et al., } \\
2013(3)(n=79)\end{array}$ & $\begin{array}{l}\text { Diab et al., } \\
2014(6)(n=27)\end{array}$ \\
\hline $\mathrm{RP}$ & $98 \%$ & $95.5 \%$ & $91.3 \%$ & NA & $92.6 \%$ \\
\hline Gl involvement & $79 \%$ & NA & $71 \%$ & NA & NA \\
\hline Esophagus dismotility & $(77 \%)$ & $72.7 \%$ & $(44.9 \%)$ & $(83.1 \%)$ & $(56 \%)$ \\
\hline Pulmonary involvement & $68 \%$ & NA & $71 \%$ & NA & NA \\
\hline ILD & $39 \%$ & $59.1 \%$ & $39.1 \%$ & $56.9 \%$ & $25.9 \%$ \\
\hline Cardiac involvement & $9 \%$ & $13.6 \%$ & $49.3 \%$ & $11.3 \%$ & NA \\
\hline Conduction alteration & NA & $16.7 \%$ & $14.5 \%$ & NA & NA \\
\hline Capillaroscopic sclerodermic pattern & NA & NA & $78.3 \%$ & $75 \%$ & $74.1 \%$ \\
\hline ANA positive & $94 \%$ & $95 \%$ & $89.9 \%$ & $100 \%$ & $94.4 \%$ \\
\hline ACA positive & $31 \%$ & $35 \%$ & $41.5 \%$ & $42.3 \%$ & $50 \%$ \\
\hline
\end{tabular}

RP, Raynaud's phenomenon; GI, gastrointestinal; NA, not available; ILD, interstitial lung disease; ANA, antinuclear antibodies; ACA, anti-centromere antibodies. 
presented by series of patients with ssSSc. Of note, none of these refer to ocular involvement in SSc (except for sicca syndrome in the Spanish Registry) or, more specifically, uveitis (3-7).

Esophagus is estimated to be involved in 70 to $80 \%$ of patients with ssSSc, which is the highest for the SSc subsets. Gastrointestinal involvement includes abdominal cramps and/or distension, nausea and vomiting, loss of weight, diarrhea, malabsorption, dysphagia and gastroesophageal reflux disease $(3,4)$. Our patient has the most characteristic feature of gastrointestinal involvement in ssSSc, which is distal esophageal dismotility. On the other hand, RP appears almost invariably in ssSSc, therefore constituting the most common symptom (4-7). Age at RP onset is variable with a mean of 42.7 years, which is earlier than skin and organ involvement (4). Nailfold capillary abnormalities are estimated to occur in about three quarters of patients with ssSSc (4-7). Our patient showed no clinical skin involvement, which is consistent with its lesser frequency in ssSSc (about 64\%) compared with other SSc subsets, except for undifferentiated SSc (4).

The largest literature review on this topic has estimated that pulmonary involvement occurs in about two thirds of patients, including dyspnea, radiologic evidence of fibrosis, $\mathrm{FVC}<70 \%$, DLCO $<70 \%$ and $\mathrm{PAH}$, whose frequency is considerably higher than that reported in $\mathrm{lcSSc}$ (11). The frequency of pulmonary fibrosis was reported to be closer to that seen for dcSSc (55 to $60 \%$ ) than for $1 \mathrm{cSSc}$ (about 20\%), whilst a recent multicenter study has shown that it is similar in ssSSc and lcSSc (around $25 \%)(4,6)$. NSIP and usual interstitial pneumonia are the most common patterns on HRCT (11). The former carries a better prognosis and should alert the clinician to the possibility of an underlying autoimmune disease, including ssSSc (11). Our patient had no PAH, although ACA were positive. Pulmonary function tests revealed a mixed pattern, which is consistent with the co-existence of COPD and interstitial lung disease.

Heart involvement is estimated to occur in about $12.5-25 \%$ of ssSSc patients, which is consistent with the prevalence seen in $\mathrm{lcSSc}$, presenting as heart failure including diastolic dysfunction, pericardial effusions, electrocardiographic abnormalities and coronary heart disease.4,10 Our patient had heart failure with severe LVSD, while left bundle branch block had been an indication to the resynchronization therapy. Moreover, cardiac fibrosis seems to be the underlying cause of a cardiomyopathy that had been previously classified as idiopathic.

Like in other subsets, more than $90 \%$ of patients with ssSSc are positive for ANA and anti-Scl-70 and ACA are the most frequent SSc-associated autoantibodies $(4,6)$. Nucleolar or speckled patterns are more specific for SSc. Diab et al. have suggested that antibody profiles of ssSSc patients are similar to those with lcSSc, including an half likelihood of having ACA and low rates of anti-topoisomerase I (16.7\% versus $11.1 \%)$ and anti-RNA polymerase III $(0 \%$ versus $7.6 \%$ ) (6). Nevertheless, the elevation of acute-phase reactants seems to be less frequent than in other variants $(4.5 \%$ versus more than $15 \%$ ) (4). Our patient had a normal C-reactive protein.

Ocular involvement in SSc often relates to scleroderma of the eyelids, including tightness of the lids and blepharophimosis (12). In a cross-sectional study of 25 patients, eyelid skin changes were present in the majority (51.1\%), keratoconjunctivitis sicca in almost half $(48.9 \%)$, cataracts in $42.2 \%$, retinal microvascular abnormalities (indistinguishable from those related to systemic hypertension and often associated with a severe capillaroscopic pattern) in $28.9 \%$, glaucoma in $13.3 \%$ and conjunctival vascular congestion in $8.9 \%$ (13). Furthermore, microcirculation seems to be affected early in the course of the disease, with significant reduction of choroidal perfusion (8). On the other hand, the frequency of eyelid skin changes is expected to be different in ssSSc patients due to the absence of skin thickening, but no prevalence studies are known. In the previous study, recurrent episcleritis was reported (4.4\%), but other inflammatory diseases, 
including scleritis, peripheral ulcerative keratitis and uveitis, were not (13). To our knowledge, there are only four case reports in the literature pointing to a rare association between SSc and uveitis in adults, since this relationship is more established in pediatric scleroderma $(9,14)$. Moreover, these cases were related to SSc with skin thickening and at least two with CREST syndrome, so this patient is exceptional, as she presents with ssSSc. Besides CAU, she also has bilateral cataracts, which are a common known complication.

In conclusion, this case underlines that $\mathrm{SSc}$ may affect several organs, even in the absence of skin involvement, so a high degree of suspicion is required when no skin thickening is present. Additionally, although other autoimmune diseases are far more common when CAU is present, one should consider the possibility of SSc when fibrosis of more than one organ is evident.

\section{REFERENCES}

1. Fett N. Scleroderma: nomenclature, etiology, pathogenesis, prognosis, and treatments: Facts and controversy. Clin Dermatol. 2013; 31: 432-7.

2. van den Hoogen, Khanna D, Fransen J, Johnson SR, Baron M, Tyndall A, et al. Classification criteria for systemic sclerosis: an American College of Rheumatology/European League against Rheumatism collaborative initiative. Arthritis Rheum. 2013; 65: 2737-47.

3. Marangoni RG, Rocha LF, Del Rio AP, Yoshinari NH, Marques-Neto JF, Sampaio-Barros PD. Systemic sclerosis sine scleroderma: distinct features in a large Brazilian cohort. Rheumatology (Oxford). 2013; 52: 1520-4.

4. Hunzelmann N, Genth E, Krieg T, Lehmacher W, Melchers I, Meurer M, et al. The registry of the German Network for Systemic Sclero- derma: frequency of disease subsets and patterns of organ involvement. Rheumatology (Oxford). 2008; 47: 1185-92.

5. Poormoghim H, Lucas M, Fertig N, Medsger T. Systemic sclerosis sine scleroderma: demographic, clinical, and serological features and survival in forty-eighty patients. Arthritis Rheum. 2000; 43: 444-51.

6. Diab S, Dostrovsky N, Hudson M, Tatibouet S, Fritzler MJ, Baron M, et al. Systemic sclerosis sine scleroderma: a multicenter study of 1417 subjects. J Rheumatol. 2014; 41: 2179-85.

7. Siméon-Aznar CP, Fonollosa-Plá V, TolosaVilella C, Espinosa-Garriga G, Ramos-Casals M, Campillo-Grau M, et al. Registry of the Spanish network for systemic sclerosis: clinical pattern according to cutaneous subsets and immunological status. Semin Arthritis Rheum. 2012; 41: 789-800.

8. Ingegnoli F, Gualtierotti R, Pierro L, Del Turco C, Miserocchi E, Schioppo T, et al. Choroidal impairment and macular thinning in patients with systemic sclerosis: the acute study. Microvasc Res. 2014; 97C: 31-6.

9. Torok KS. Pediatric scleroderma - systemic and localized forms. Pediatr Clin North Am. 2012; 59: 381-405.

10. Fischer A, Meehan R, Feghali-Bostwick C, West S, Brown K. Unique characteristics of systemic sclerosis sine scleroderma-associated interstitial lung disease. Chest. 2006; 130: 976-81.

11. Toya SP, Tzelepis GE. The many faces of scleroderma sine scleroderma: a literature review focusing on cardiopulmonary complications. Rheumatol Int. 2009; 29: 861-8.

12. Thorne JE, Jabs DA. Ocular manifestations of the rheumatic diseases. In: W. Tasman, ed. Duane's clinical ophthalmology. Vol. 5. Philadelphia, PA: Lippincott Williams \& Wilkins; 2005; 22-4.

13. Gomes BAF, Santhiago MR, Magalhães P, Kara-Junior N, Azevedo MNL, Moraes Jr. $\mathrm{HV}$. Ocular findings in patients with systemic sclerosis. Clinics. 2011; 66: 379-85.

14. Bolad W. Anterior uveitis in a patient with scleroderma: a case report [Abstract]. Ocul Immunol Inflamm. 2013; 21: 254. 